Ann. Biol. anim. Bioch. Biophys., 1979, 19 (2-A), 445-465.

\title{
L'organe pinéal du Brochet (Esox lucius, L.). I. Etude anatomique et cytologique
}

\author{
par J. FALCON *
}

\begin{abstract}
Laboratoire de Zoologie ef Biologie cellulaire ef Laboratoire associé au CNRS no 290

40, Avenue du Recteur Pineau, 86022 Poitiers Cedex, France.
\end{abstract}

Summary. The pineal organ of the pike, Esox lucius L., I. A light and electron microscopic study.

This report presents a study of the pineal organ of pike using light and electron microscopy. Light microscopy showed a clear regional differentiation. The pineal end-vesicle was composed of the saccular distal pineal and the middle pineal with a folded and wellvascularized epithelium ; the caudal pineal (or pineal stalk) connected the pineal endvesicle to the diencephalic roof. Tissues overlying the pineal organ appeared to be specialized for light penetration. The pineal epithelium was composed of (i) more or less well differentiated photoreceptor cells basically resembling retinal photoreceptors, (ii) supporting cells, and (iii) sensory neurons with an axon reaching the pineal tract. Bundles of unmyelinated axons were seen near the lateral walls of the rostral part of the pineal stalk ; in the caudal part they converged dorsally to form the pineal tract composed of myelinated and unmyelinated fibers. A bundle of axons was also identified over the pineal stalk. The general pineal organization suggested a photosensory function. A preliminary electron microscopic study showed supporting cells and a fourth cell type (with undetermined inclusions) of the sensory pineal epithelium ; their possible functions are discussed. The components of the middle pineal lumen were characterized by an accumulation of material (probably outer segments of degenerating photoreceptor cells) and the presence of free phagocytotic cells (macrophages). The pineal organ was surrounded by a connective tissue layer, richly supplied with fenestrated capillaries in the middle region, suggesting important exchanges. Some sympathetic-like nerve terminals were also found in the perivascular spaces of the middle pineal. No definite evidence of a secretion was evidenced in the present study, but this negative observation in no way excluded neuroendocrine function. Further studies using refined techniques must prove or disprove whether a functional differentiation corresponds to the morphohistological regional differentiation.

\section{Introduction.}

Les études morphologiques effectuées sur l'épiphyse des Poissons Téléostéens ont conduit, très tôt, les auteurs à suggérer les adaptations de l'organe à la photoréception (Studnicka, 1905 ; Hafeez et Ford, 1967 ; Omura ef Oguri, 1969 ; Hafeez, 1971). Les recherches ultrastructurales corroborent les données anatomiques (Salmo: Breuc-

* Max-Planck-Institut für Physiologische und Klinische Forschung, W. G. Kerckhoff-Institut, Parksirasse 1, 6350 Bad-Nauheim, BRD. 
ker et Horstmann, 1965 ; Hartwig et Pfautsch, 1973 ; Mugil : Rüdeberg, 1966 ; Uranoscopus : Rüdeberg, 1966 ; Sardina : Rüdeberg, 1968 ; Phoxinus : Oksche et Kirschstein, 1967, 1971 ; Carassius : Takahashi, 1969 ; Scyliorhinus : Rüdeberg, 1969 ; Plecoglossus : Omura et al., 1969 ; Omura et Oguri, 1971 ; Esox : Owman et Rüdeberg, 1970 ; Anguilla et Lebistes : Rüdeberg, 1971 ; Poecillia : Omura et Oguri, 1971 ; Thunnus : Murphy, 1971 ; Pterophyllum : Bergmann, 1971 ; Oryzias : Takahashi ef Kasuga, 1971 ; Urasaki, 1976a ; Astyanax : Omura, 1975 ; Herwig, 1976 ; Bathylagus et Nezumia : McNulty, 1976 ; Triphoturus : McNulty et Nafpaktitis, 1976 ; Latimeria : Hafeez et Merhige, 1977). Une fonction photosensorielle a d'ailleurs été démontrée électrophysiologiquement chez quelques-unes des espèces étudiées en microscopie électronique (Salmo : Dodt, 1963 ; Morita, 1966 ; Tabata et al., 1975 ; Salmo, Plecoglossus, Mugil, Pelteobagrus : Hanyu ef al., 1969 ; Hanyu et Niwa, 1970 ; Pterophyllum : Morita et Bergmann, 1971).

L'organe pinéal des Téléostéens présente également une activité neuroendocrinienne puisqu'il agit - sous l'influence des facteurs lumineux - sur l'activité motrice et la pigmentation (Salmo : Hafeez, 1970 ; Hafeez et Quay, 1970a ; Carassius : Fenwick, 1970a), ainsi que sur le développement des gonades (Carassius : Fenwick, 1970b ; Oncorhynchus : Fenwick, 1970c ; Fundulus : de Vlaming et al., 1974 ; Oryzias : Urasaki, 1976b) ; cette activité est réalisée par l'intermédiaire de la mélatonine (Fenwick, 1970c ; Hafeez, 1970). Dans l'épithélium épiphysaire des Téléostéens, ont d'ailleurs été mis en évidence des composés de nature indolique - dont la mélatonine - (Salmo : Oguri ef al., 1968 ; Hafeez et Quay, 1969 ; Hafeez et Zerihun, 1976 ; Atherinopsis : Hafeez et Quay, 1969 ; Esox : Owman et Rüdeberg, 1970 : Oncorhynchus : Fenwick, 1970c) ainsi que les enzymes impliquées dans la synthèse de la mélatonine (Salmo : Quay, 1965 ; Salmo ef Hesperoleucus : Hafeez et Quay, $1970 b$; Salmo : Smith et Weber, $1976 a$ et $b$ ).

Mais aucun auteur n'a réussi chez les Poissons, comme d'ailleurs chez tous les autres Anamniotes, à identifier le (ou les) type(s) cellulaire(s) qui élabore(nt) les principes actifs. En fait, il s'agit de résoudre l'un des problèmes importants de physiologie cellulaire posés par Collin $(1969,1971)$ : un photorécepteur typique est-il capable d'élaborer deux types de messages, l'un sensoriel, l'autre neurohormonal (mélatonine et éventuellement d'autres dérivés indoliques) ? Contrôle-t-il directement et selon quelles modalités la synthèse et/ou la libération de tels dérivés indoliques ? Ou bien alors, la biosynthèse des composés indoliques a-t-elle lieu, comme chez les Sauropsidés, au niveau de photorécepteurs rudimentaires? Dans cette éventualité, il faut supposer la mise en place d'une rudimentation chez certains Anamniotes : celle-ci, déjà suggérée par Collin $(1969,1977)$, n'a pas encore vraiment retenu l'attention des auteurs.

Owman et Rüdeberg (1970) ont mis en évidence les précurseurs de la mélatonine (5-hydroxytryptophane ; sérotonine) dans l'organe pinéal du Brochet (Esox lucius, L.), considéré comme photosensoriel. Néanmoins l'étude de cette espèce, pas plus que celle des autres genres, n'a permis aux auteurs de répondre aux problèmes posés cidessus. De plus, Owman et Rüdeberg (1970) concluent à l'activité photosensorielle, uniquement sur la mise en évidence ultrastructurale de certains caractères des cellules de type photorécepteur ; ils ne parviennent pas à observer le système de neurones impliqués dans la conduction des messages sensoriels d'origine photique. 
L'épiphyse des Poissons, à l'issue des études préliminaires, apparaît comme un organe relais, capable de capter certaines des informations de l'environnement ef d'élaborer des messages susceptibles de participer au contrôle, selon des voies encore inconnues, d'effecteurs variés. A l'échelle cellulaire, l'organe pinéal des Poissons offre donc un modèle d'étude original et intéressant dans le domaine des interactions. Néanmoins, une connaissance approfondie de son architecture doit précéder une telle étude physiologique.

Le Brochet nous a paru, à la suite des recherches d'Owman et Rüdeberg (1970), représenter un matériel d'étude favorable. Il est relativement commun dans les rivières et étangs de notre région (Poitou-Charentes et Berry). De plus, les mécanismes de contrôle de l'axe hypothalamo-hypophyso-gonadique par l'organe pinéal, présente, en relation avec l'environnement chez Esox, comme chez tous les Téléostéens, un intérêt économique évident. Nous présentons ici les résultats de notre étude anatomique et cytologique préliminaire.

\section{Matériel et méthodes.}

1) Matériel biologique. — Les Brochets (Esox lucius, L.) fraîchement capturés nous ont été fournis, d'octobre à février, par la pisciculture du Moulin de Chauvineau (Quinçay, Vienne) et la Station d'acclimatation et d'Elevage (Stacel : Argenton-l'Eglise, Deux-Sèvres). Leur longueur varie de 20 à $40 \mathrm{~cm}$. Ils sont transportés dans des sacs en matière plastique transparente, partiellement remplis d'eau et gonflés à l'oxygène (la durée du transport n'excède pas $1 \mathrm{~h}$ ). A leur arrivée au laboratoire, les animaux sont placés dans des aquariums de $400 \mathrm{I}$, à circulation continue et munis de diffuseurs d'air. Ils sont éventuellement nourris de proies vivantes (Vairons, Gardons, Carpeaux) et soumis au rythme photopériodique naturel. Certains exemplaires sont gardés 3 à 4 semaines au plus.

2) Prélèvement de l'épiphyse ef de l'encéphale. - Les animaux sont sacrifiés par décapitation. Un volef osseux est découpé au-dessus de l'encéphale qui est immédiatement arrosé de fixateur. Le prélèvement intéresse soit l'épiphyse seule (en $3 \mathrm{~min}$ ), soit l'ensemble de l'encéphale (en $5 \mathrm{~min}$ ) lorsque doivent être conservées les connexions avec la voûte diencéphalique.

3) Techniques histologiques ef cytologiques.

Etude histologique (Martoja et Martoja, 1967). - 6 encéphales de Brochet ont été fixés in fofo par immersion dans le Bouin-Hollande-Sublimé (BHS), puis déshydratés par les alcools et inclus dans la paraffine. Les blocs sont débités en coupes parasagittales de $7 \mu \mathrm{m}$ d'épaisseur qui sont étalées sur lames de verre, colorées à l'hémalunéosine et montées dans le DPX (mélange de Distrène, Phtalate de butyle et Xylène).

Elude cyfologique. - 14 Brochets ont été utilisés. Les épiphyses sont fixées de $30 \mathrm{mn}$ à $4 \mathrm{~h}$ par immersion dans le glutaraldéhyde (Fluka) à 2 ou 2,5 p. 100 dans le tampon phosphate selon Sörensen $(0,1 \mathrm{M} ; \mathrm{pH} 7,4)$. Le temps de fixation varie de $30 \mathrm{mn}$ à $4 \mathrm{~h}$. Après 3 rinçages d'une $1 / 2 \mathrm{~h}$ dans le même tampon, elles sont postfixées par le tétroxyde d'osmium $\left(\mathrm{OsO}_{4}\right)$ à 2 p. 100 dans le tampon Sörensen pendant 
$1 \mathrm{~h}$. La déshydratation est effectuée par bains successifs dans l'alcool éthylique de degré croissant $\left(30^{\circ}\right.$ à $\left.100^{\circ}\right)$. Après passage dans l'oxyde de propylène, les pièces sont placées une nuit dans le mélange à volume égal oxyde de propylène et Epon ; elles sont éventuellement fractionnées en 3 parties : distale, moyenne et proximale, puis sont incluses dans l'Epon 812 (Luft, 1961).

Pour l'étude cytologique, en microscopie photonique, des coupes transversales de $1 \mu \mathrm{m}$ d'épaisseur sont réalisées sur un ultramicrotome Reicherf OMU 2, puis étalées sur lames de verre et colorées au bleu de toluidine.

En alternance avec les coupes semi-fines, des coupes ultrafines sont recueillies sur grilles de cuivre de 300 mesh et doublement contrastées à l'acétate d'uranyle en solution saturée (Watson, 1958) et au citrate de plomb (Reynolds, 1963). Elles sont examinées au microscope électronique Hitachi HU $11 \mathrm{Cs}$, sous une tension de $75 \mathrm{kV}$.
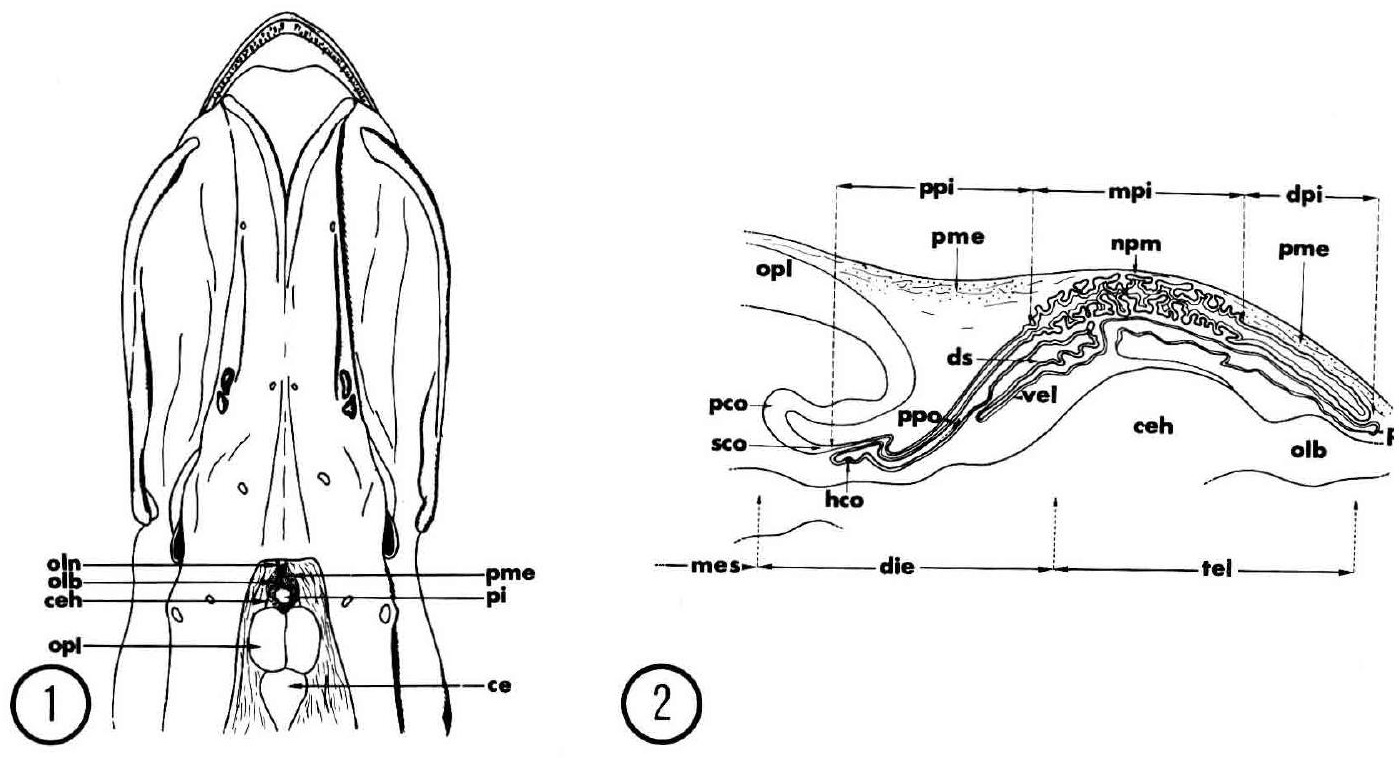

PLANCHE I

FIG. 1. - Schéma de la tête de Brochet en vue dorsale, montrant l'organe pinéal in situ.

FIG. 2. - Schémo de la région du diencéphale en coupe sagiftale. ce : cervelet (cerebellum) ; ceh : hémisphères cérébraux (cerebral hemispheres); die : diencéphale (diencephalon); $d p i$ : épithélium épiphysaire de la région distale (distal pineal); ds : sac dorsal (dorsal sac); hco : commissure habénulaire (habenular commissure); mes : mésencéphale (mesencephalon); mpi : épithélium épiphysaire de la région moyenne (middle pineal) ; npm : méninges non pigmentées (non pigmented meninges) ; olb : bulbe olfactif (olfactory bulb) ; oln : nerf olfactif (olfactory nerve) ; opl : lobe optique (optic lobe) ; par : paraphyse (paraphysis) ; pi : organe pinéal (pineal organ) ; pco : commissure postérieure (posterior commissure); pme : méninges pigmentées (pigmented meninges) ; ppi : épithélium épiphysaire de la région proximale (proximal pineal) ; ppo : organe parapinéal (parapineal organ); sco: organe sous-commissural (sub-commissural organ); tel : télencéphale (telencephalon); vel : vélum transverse (velum transversum). 


\section{Résultats.}

1) Etude anatomique. - L'épiphyse du Brochet, vésicule aplatie, recouvre le télencéphale dans sa totalité ainsi que les bulbes olfactifs (fig. 1,2). Elle est reliée au diencéphale par un fin pédoncule ou tige pinéale, dans une région comprise entre la commissure habénulaire et la commissure postérieure. La tige pinéale est percée d'un étroit canal qui fait communiquer la lumière de la vésicule avec le $3^{e}$ ventricule (fig. 2,3 ).

Notre éfude nous a conduit à distinguer 3 régions :

a) Région distale ou rostrale (fig. 1-3) : elle est recouverte par des méninges fortement pigmentées. L'épithélium pinéal présente une surface plane ou très légèrement plissée : il est plus épais ventralement que dorsalement.

b) Région intermédiaire ou moyenne (fig. 2,3) : les méninges qui la recouvrent ne sont pas pigmentées; l'épithélium est plissé et très richement vascularisé.

Les régions distale et moyenne forment la vésicule épiphysaire.

c) Région proximale ou caudale ou fige épiphysaire (fig. 2,4$)$ : elle est recouverte de méninges fortement pigmentées. Son épithélium, assez semblable à celui de la région distale, est cependant moins épais. La tige épiphysaire est logée dans une gouttière formée par les replis du sac dorsal (fig. 9).

2) Efude, en microscopie photonique, des types cellulaires de l'épithélium sensoriel. 3 types cellulaires peuvent être identifiés sur les coupes semi-fines colorées au bleu de toluidine.

a) Cellules de type photorécepteur (CTP) (fig. 5). Elles occupent presque toute la hauteur de l'épithélium épiphysaire. Généralement, elles présentent une affinité particulière pour le colorant. On peut reconnaître les 4 régions fondamentales d'une cellule photoréceptrice typique soit :

- un segment externe qui encapuchonne la partie apicale du segment interne sousjacent. Mais certaines CTP semblent en être dépourvues;

- un segment interne constitué de 2 parties : une région apicale, qui fait saillie ou non dans la lumière, reliée à une région proximale par un étranglement (ou cou cellulaire) plus ou moins prononcé. Les différentes régions du segment interne sont particulièrement riches en inclusions en forme de bâtonnets (correspondant aux mitochondries);

— un corps cellulaire qui prolonge le segment interne; le noyau possède un nucléole excentrique;

- un (des) pédicule(s) basal(aux) : issu(s) du corps cellulaire et se dirigeant vers la lamelle basale ; son (leur) trajet exact est difficile à suivre.

Dans certaines CTP (notamment dans la région moyenne), on note la présence de vacuoles bien développées (fig. 6). II s'agit de cellules en dégénérescence (Falcon, 1979).

b) Cellules interstitielles de type épendymaire (CIE) (fig. 5). Situées entre les CTP, elles sont moins intensément colorées que ces dernières et occupent toute la hauteur 


\section{PLANCHE II}

Histo- et cytologie de l'organe pinéal d'Esox lucius $L$.

FIG. 3 et 4. - Vésicule ef tige épiphysaires. Coupes parasagittales colorées à l'hémalun-éosine. Figure 3: L'épithélium de la partie distale (dpi) est peu plissé par rapport à celui de la région moyenne (mpi) $(\times 58)$. Figure 4: L'épithélium de la tige épiphysaire, peu vascularisé, n'est pas plissé. La lumière de l'organe ( $p l)$ communique avec le $3^{\mathrm{e}}$ ventricule (III) (ca : capillaire ; $d s$ : sac dorsal ; heo : commissure habénulaire ; par : paraphyse ; sco : organe sous-commissural) $\times 50$.

FIG. 5. - Cellules de type photorécepteur ( $p$ ) et cellules inferstitielles (ic). Coupe semi-fine colorée au bleu de toluidine. On reconnaît aisément les 4 parties fondamentales des cellules de type photorécepteur (voir texte). ( $c a:$ capillaire ; $c b$ : corps cellulaire; $c n:$ cou cellulaire ; is : segment interne ; os : segment externe ; pp : pédicule basal) $(\times 660)$.

FIG. 6,7 et 8. - Neurones $(p n)$ et groupes d'axones dans la vésicule épiphysaire. Coupes semi-fines colorées au bleu de toluidine. Figure 6: Neurone de la région distale (pn), localisé dans l'épithélium basal (os : segments externes de photorécepteurs) ( $\times$ 640). Figure 7: Un neurone, dans la partie caudale de la région moyenne, montre le départ d'une expansion dendritique (dd) ainsi que celui d'un axone $(a x)$ qui se dirige vers un groupe de fibres localisées dans l'épithélium basal (petites flèches) $(\times 380)$. Figure 8: Groupes d'axones (flèches) dans la région moyenne, convergeant vers la région proximale $(\times 210)$.

FIG. 9 et 10. - Tige épiphysaire. Coupes transversales colorées au bleu de folvidine. Figure 9: Zone médio-antérieure avec plusieurs groupes de fibres dans l'épithélium épiphysaire (flèches) ; quelques rares fibres sont myélinisées $(m n)$; un faisceau de fibres myélinisées et nues est visible au-dessus de la tige épiphysaire (ef) ( $d s$ : sac dorsal) $(\times 200)$. Figure 10: Zone postérieure ; le tractus pinéal ( $p t r)$ est composé d'un faisceau unique médio-dorsal ; les fibres myélinisées sont nombreuses (ca : capillaire ; $p l$ : lumière épiphysaire) $(\times 295)$. 
de l'épithélium. Le noyau, également moins chromophile, est situé à la partie basale de la cellule.

c) Neurones sensoriels (NS) (fig. 6, 7). Difficiles à identifier sur les coupes semi-fines colorées au bleu de toluidine, ils sont foujours localisés dans le tiers basal de l'épithélium. Ils possèdent un noyau volumineux, sphérique ; le nucléole excentrique, également sphérique, est très chromophile (fig. 6). Le cyłoplasme très peu coloré, présente parfois un aspect vacuolaire (fig. 6). Les départs des prolongements axoniques et/ou dendritiques sont visibles sur certaines préparations. L'axone se dirige généralement vers les faisceaux de fibres (= axones d'autres neurones) situés près de la lamelle basale (fig. 7 ; voir également ci-dessous).

d) Tige épiphysaire (fig. 8-10). On peut observer, dès la région moyenne, aussi bien dans l'épithélium dorsal que ventral, des groupes de fibres nerveuses situés près de la lamelle basale (fig. 7). Ces faisceaux sont vraisemblablement constitués par les axones des neurones sensoriels de la région distale. La tige présente, sur toute sa longueur, un faisceau médio-dorsal, accompagné dans sa région proximale antérieure de faisceaux latéraux souvent moins développés et en nombre variable : 1 d̀ 5 (fig. 8 , 9). Ces derniers rejoignent progressivement le faisceau médio-dorsal pour constituer un tractus unique dans la région proximale postérieure (fig. 10).

Dès la partie moyenne du tractus, certaines fibres s'entourent d'une gaine de myéline (fig. 9). Le nombre de fibres myélinisées augmente au fur et à mesure que l'on

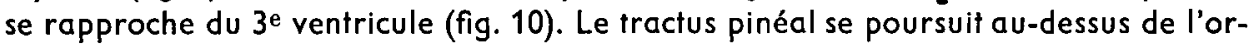
gane sous-commissural, en direction de la commissure postérieure (Falcon et Mocquard, 1979).

Des groupes d'axones (myélinisés ou nus) sont également observés au-dessus de la tige épiphysaire, dans sa région la plus caudale (fig. 9). Leur signification sera discutée ultérieurement.

Dans la lumière épiphysaire, des débris cellulaires ainsi que des cellules (très vraisemblablement des macrophages) sont observés (voir paragraphe 4).

\section{PLANCHE III}

\section{Uitrastructure des cellules interstitielles}

FIG. 11. - Epithélium épiphysaire d'Esox lucius L. A côté de 2 cellules de type photorécepteur (P), une cellule interstitielle (IC) occupe toute la hauteur de l'épithélium (voir également Falcon, 1979, fig. 1). Le noyau (n) est localisé dans la partie basale de la cellule plus large qui envoie de nombreux prolongements (icp). La partie moyenne est mince. La cellule s'élargit à l'apex qui présente ici 2 corps résiduels $(r b)$ (bl: lamelle basale; $c b$ : corps cellulaire; $c n$ : cou cellulaire ; $g$ : appareil de Golgi ; is : segment interne ; $m$ : mitochondries; $m f$ : microfilaments ; pp : pédicule de photorécepteur).

Dans la lumière épiphysaire $(p l)$, on remarque de nombreuses structures membranaires désorganisées $(\times 7800)$.

FIG. 12. - Apex d'une cellule interstitielle (IC). Parmi les nombreuses microvillosités ( $m v$ ), on remarque dans la lumière, une structure ciliaire de type $(9+1)(c i)$. L'apex de la cellule présente un centriole axial $\left(c_{1}\right)$ et un centriole distal $\left(c_{2}\right)(\times 32000)$. 
3) Etude, en microscopie électronique, des types cellulaires apparemment non directement impliqués dans la phoforéception ef la transmission des messages sensoriels. - Compte tenu de l'intérêt touf particulier que présentent les cellules de type photorécepteur et les neurones sensoriels, une étude séparée leur est consacrée (Falcon, 1979; Falcon et Mocquard, 1979). Seront examinées seulement ici :

a) Cellules interstitielles de type épendymaire (fig. 11). Les cellules interstitielles (ou cellules de soutien d'autres auteurs) entrent toujours en confact avec la lamelle basale de l'épithélium épiphysaire et bordent, à leur partie apicale (fig. 11, 12), la lumière épiphysaire. Le noyau est localisé dans la région centrale ou basale, plus large. A ce niveau, la cellule envoie de nombreux prolongements ramifiés. Dans certaines parties de l'épithélium basal (région intermédiaire en particulier), on observe uniquement de nombreux prolongements grêles qui "prennent appui » sur la lamelle basale. La cellule est grêle dans sa partie moyenne, plus large en bordure de lumière où elle présente généralement des microvillosités. A ce niveau peuvent être identifiées des structures centriolaires et ciliaires de type $9+1$ ( 9 doublets périphériques +1 doublet central) (fig. 12).

Des microtubules et des microfilaments sont orientés selon le grand axe de la cellule. Les mitochondries, cupuliformes ou en bâtonnets (parfois géantes), sont réparties dans toute la cellule. Leur matrice est relativement dense; les tubules internes ne sont pas dilatés. Le glycogène est peu abondant (Falcon, 1979). On observe quelques lysosomes, des cytosomes (présentant parfois des inclusions lipidiques), des vacuoles digestives ou des inclusions à corps denses (corps résiduels).

Les organites, impliqués dans les synthèses (protéiques et glycoprotéiques), sont bien développés dans la majorité des cas. L'ergastoplasme se présente sous la forme, soit de vésicules et canalicules interconnectés, soit de citernes parfois très développées occupant ainsi un important volume dans la cellule (fig. 13, 14). Les citernes sont rem-

\section{PLANCHE IV}

Ultrastructure des cellules interstitielles ef des constifuants de la lumière épiphysaire

FIG. 13. - Dans les cellules inferstitielles (IC), l'ergastoplasme (rer) se présente le plus souvent sous la forme de citernes dilatées, remplies d'un matériel finement granulaire, peu dense aux électrons ( $P$ : cellule de type photorécepteur) $(\times 15000)$.

FIG. 14. - Citerne géante de l'ergastoplasme dans une cellule interstitielle $(\times 25000)$.

FIG. 15. - Appareil de Golgi $(g)$ et réticulum endoplasmique lisse (ser) dans une cellule inferstitielle ( $m$ : mitochondrie) $(\times 22000)$.

FIG. 16. - Cellule à inclusions indéferminées. Au voisinage de l'appareil de Golgi (g), bien développé, on remarque (petites flèches) de nombreuses vésicules claires. Les inclusions de 1300 à $3900 \AA$ de diamètre (têtes de flèches) constituent la caractéristique essentielle ( $m f:$ microfilaments) $\times 25000$ )

FIG. 17. - Lumière épiphysaire dans la région moyenne. On note une accumulation importante de structures membranaires, correspondant très vraisemblablement à des segments externes désorganisés (dos) en dégénérescence (is : segment interne d'une cellule de type photorécepteur) $(\times 6400)$. 
plies d'un matériel finement granulaire, peu dense aux électrons. Le réticulum lisse occupe également d'importantes régions dans la cellule ; il est uniquement formé de petites vésicules ef de canalicules (fig. 15). Les dictyosomes sont composés de saccules parfois dilatés qui montrent des relations avec le réticulum (lisse en particulier). Les ribosomes libres sont nombreux (fig. 15).

b) Cellules à inclusions «indéterminées » $(\mathrm{CII})$. La microscopie électronique révèle l'existence d'une catégorie de cellules différentes, à plusieurs points de vue, des 3 autres types cellulaires de l'épithélium épiphysaire.

Peu nombreuses, elles sont caractérisées par une accumulation importante d'inclusions généralement sphériques ou ovoïdes, variant très largement en taille (1 300 à $3900 \AA$ de diamètre). Elles sont délimitées par une structure membranaire, parfois mal visualisée. Elles contiennent ou non un matériel finement granulaire moyennement dense aux électrons ; dans tous les cas, un halo sombre, périphérique, est visible à l'intérieur (fig. 16).

Ces cellules sont toujours siłuées dans la partie basale de l'épithélium, leur axe longitudinal orienté parallèlement à la lamelle basale. Souvent, elles semblent encapsulées par les prolongements des cellules interstitielles voisines, de telle sorte que des contacts avec les CTP ne sont jamais observés. Outre les inclusions citées, le cytoplasme contient quelques mitochondries, des microtubules et des faisceaux de microfilaments (fig. 16). Le glycogène est peu abondant. Les dictyosomes sont constitués, chacun, par un empilement de 4 à 5 saccules, souvent dilatés. Les saccules libèrent à leur périphérie des vésicules claires de 650 à $1100 \AA ̊$ de diamètre (fig. 16). Contrairement à l'ergastoplasme, le réticulum agranulaire est bien développé et constitué de vésicules et canaux interconnectés qui entrent parfois en relation avec l'appareil de Golgi (fig. 16).

Le noyau est caractérisé par une chromatine granulaire irrégulièrement répartie (plus dense à la périphérie) ; le nucléole n'a jamais été observé.

4) La lumière épiphysaire ef la région périépiphysaire : étude en microscopie électronique.

a) La lumière épiphysaire. Dans la lumière épiphysaire s'observent de nombreux segments externes et structures ciliaires, appartenant aux cellules de type photorécepteur (Falcon, 1979). On remarque également, en particulier dans la région moyenne, une accumulation importante de structures membranaires polymorphes (fig. 17). II s'agit très probablement de segments externes en dégénérescence, qui ne sont plus rattachés aux cellules à partir desquelles ils se sont différenciés (Falcon, 1979). Ces siructures semblent être digérées par les CIE (voir précédemment) et surtout par les nombreuses cellules phagocytantes $(C P)$, libres dans la lumière $(=$ macrophages des différents auteurs : des Cyclostomes aux Oiseaux) (fig. 18, 19).

Le noyau polymorphe de ces cellules possède une chromatine dense et granulaire. Dans le cytoplasme, réticulum lisse el granulaire sont constitués de saccules dilatés ou allongés. De nombreux ribosomes libres forment des polysomes. Le complexe golgien est particulièrement développé ; les dictyosomes paraissent reliés entre eux par un réseau complexe de canalicules (fig. 19) ; leurs saccules sont dilatés. A proximité, on remarque de nombreuses vésicules à contenu plus ou moins dense (750 à $1200 \AA$ de diamètre) ainsi que des lysosomes ( 2300 à $2800 \AA$ de diamètre). Dans le cytoplasme, 
se rencontrent également de nombreuses vacuoles digestives de taille très variable (fig. 18, 19). Le matériel en digestion est d'origine diverse ; on remarque fréquemment des structures rappelant des segments externes plus ou moins désorganisés (fig. 19). Cerłaines électronographies suggèrent que le matériel en dégénérescence est capté par phagocytose (fig. 18).

b) La région périépiphysaire. L'épiphyse est limitée sur toute sa périphérie par une lamelle basale (200 à $300 \AA$ d'épaisseur) séparant son épithélium des espaces péricapillaires (fig. 20).

La périphérie de la vésicule épiphysaire (régions moyenne + distale) est richement vascularisée : l'endothélium des capillaires (fig. 20, 21) présente de nombreuses fenestrations fermées ou non par une structure membranaire ou diaphragme; on observe également quelques vésicules d'endocyłose. L'espace périvasculaire (espace compris entre les lamelles basales de l'endothélium capillaire et de l'épithélium épiphysaire) est peu important. Dans la région proximale, les capillaires sont moins nombreux (cf. résultats anatomiques). A ce niveau, l'endothélium des capillaires n'est pas fenêtré ; il est plus épais et l'espace périvasculaire plus important.

Dans l'espace périvasculaire, on trouve des fibrilles de collagène, quelques fibrocyłes et des péricytes juste à proximité des capillaires (fig. 20, 21).

Exceptionnellement, nous avons observé des sections transversales d'axones (fig. 22) qui, par leur contenu (nombreuses vésicules claires de 320 à $640 \AA$ de diamètre et rares vésicules à cœur dense de diamètre plus élevé), rappellent les fibres orthosympathiques de l'épiphyse des Amniotes.

\section{Discussion.}

L'étude en microscopie photonique seule, région par région, par la technique des coupes sériées apporte des compléments substantiels à celle d'Owman et Rüdeberg (1970).

Les critères anatomiques sont en faveur d'une activité photosensorielle de l'organe. La vésicule épiphysaire, accolée à la voûte crânienne, est parfaitement visible par transparence (donc aisément atteinte par la lumière). Les pigments, dans les méninges qui circonscrivent l'organe pinéal, présentent une distribution zonée ef pourraient, en fonction de leur répartition, contrôler la lumière incidente.

Les études d'Omura et Oguri (1969) et de Hafeez (1971) montrent qu'il existe des variations histologiques importantes d'une espèce à l'autre. Ainsi, chez certains Poissons, l'organe pinéal est tubulaire, l'épithélium n'est pas plissé, sa lumière communique avec le 3 e ventricule; chez d'autres, la lumière disparaît, l'épithélium devient « compact », le nombre de cellules photoréceptrices semble être réduit. Tous les stades intermédiaires sont observés entre ces 2 types. Selon les auteurs ci-dessus, le passage d'un type à l'autre pourrait traduire le développement d'une activité glandulaire aux dépens de la fonction sensorielle. Contrairement à Owman ef Rüdeberg (1970), nous avons remarqué une «zonation 》 morphologique dans l'épithélium épiphysaire d'Esox. En fait, le développement de l'épithélium plissé et très vascularisé dans la région moyenne contraste avec celui des régions distale et proximale. Comple tenu de l'interprétation d'Omura et Oguri (1969) et d'Hafeez (1971), la structure de l'épiphyse 


\section{PLANCHE V}

Ultrastructure des constituants de la lumière épiphysaire ef de la région périépiphysaire.

FIG. 18 ef 19. - Cellules phagocytantes ou macrophages (MA) dans la lumière épiphysaire. Figure 18: Cette électronographie suggère un capłage du matériel en dégénérescence par phagocyłose (têtes de flèches). On remarque la présence de nombreuses vacuoles digestives (dva) et des corps résiduels $(r b)(\times 6700)$. Figure 19: L'appareil de Golgi $(g)$ est bien développé ; des canalicules (petites flèches) semblent relier les dictyosomes entre eux. On note, au voisinage, la présence de lysosomes $(l y)$. Les vacuoles digestives $(d v a)$ contiennent très fréquemment des structures membranaires concentriques $(m w)(\times 14500)$ (dos : segments externes désorganisés, dans la lumière épiphysaire ; rer : ergastoplasme).

FIG. 20. - Espace périvasculaire dans la région moyenne de l'épiphyse. Organe pinéal et endothélium (e) des capillaires (ca) sont délimités, à la périphérie, par une lamelle basale (bl). Dans l'espace périvasculaire, on rencontre de nombreuses fibrilles de collagène ( $f f$ ). L'endothélium (e) des capillaires $(c a)$ présente de nombreuses fenestrations (petites flèches) diaphragmées (ER : érythrocyte) $(\times 27000)$.

FIG. 21. - Endothélium (e) d'un capillaire (ca) dans la région proximale. Contact entre deux cellules endothéliales (cercle) (PC : péricyte) ( $\times 69000)$.

FIG. 22. - Terminaisons axoniques (at) dans la région périépiphysaire, contenant de nombreuses vésicules claires (petites têtes de flèches) et quelques vésicules à cœur dense (têtes de flèches larges) ( $F$ : fibrocyte ; $m$ : mitochondrie) $(\times 48000)$. 
moyenne d'Esox évoque-t-elle la mise en place d'une activité glandulaire aux dépens de la fonction sensorielle?

L'adaptation de l'endothélium (type fenestré) des capillaires aux échanges avec l'épithélium de la région moyenne et l'existence de terminaisons vraisemblablement orthosympathiques dans l'espace périvasculaire contigu (susceptible de contrôler les synthèses indoliques; Quay, 1974) constituent à elles seules des critères encore très insuffisants pour illustrer un changement éventuel de fonction.

Des différences régionales assez voisines de celles de l'organe pinéal du Brochet, ont été rapportées chez un autre Téléostéen (Engraulis : Hafeez,1971).

Les recherches cytophysiologiques et expérimentales complémentaires devrajent permettre d'établir si à la «zonation » morphologique proposée ici, correspond réellement une «zonation » fonctionnelle.

Les analogies cytologiques entre les CTP épiphysaires et les photorécepteurs de la rétine des yeux latéraux, de même que la présence de neurones sensoriels permettent également de suggérer l'existence d'une fonction photosensorielle (voir discussion in Collin, 1969) chez Esox. Selon les régions considérées, les CTP montrent en microscopie photonique, de façon plus ou moins distincte, leurs segments externes. Compte tenu du polymorphisme de ces articles, de leur taille variable, d'une rudimentation possible (Collin, 1969-1977) et d'un éventuel renouvellement partiel ou in toto des empilements de disques, seule une étude détaillée en microscopie électronique (Falcon, 1979) peut permettre d'évaluer le stade de différenciation des segments externes (ou pôles photorécepteurs) et des cellules de type photorécepteur d'une façon générale. La présence de macrophages, impliqués dans les mécanismes de phagocytose et de digestion de débris cellulaires et structures en dégénérescence, a déjà été signalée dans la lumière épiphysaire de certains Poissons (Carassius : Takahashi, 1969 ; Anguilla : Rüdeberg, 1971 ; Astyanax : Omura, 1975 ; Herwig, 1976), Batraciens (Rana : Bunt ef Kelly, 1971 ; Diemictylus : Flight, 1973) ef Sauropsidés (Collin, 1969).

Contrairement à Owman ef Rüdeberg (1970), nous avons observé quelques neurones sensoriels. Mais, si toutes les fibres du tractus appartiennent à l'innervation sensorielle pinéalofuge (comme chez Salmo : Hafeez et Zerihun, 1974), leur nombre élevé n'a cependant pas de rapport avec la rareté des corps cellulaires des neurones sensoriels observés. Deux explications peuvent être avancées : 1) les neurones sensoriels sont $\mathrm{mal}$ visualisés par les méthodes classiques d'inprégnation ou de coloration (Rüdeberg, 1970 ; Hafeez, 1971 ; McNulty, 1976 ; McNulty ef Nafpaktitis, 1976) ef/ou 2) ils sont uniquement groupés dans certaines zones de l'épithélium (Collin, 1969). La présence de groupes d'axones dans la région intermédiaire corrobore la seconde probabilité. Le problème du tractus pinéal sera abordé ultérieurement (Falcon, 1979 ; Falcon el Mocquard, 1979). Notons ici, que l'existence de faisceaux de fibres à l'extérieur de la lamelle basale au-dessus de la tige épiphysaire ne peut encore être interprétée. Il pourrait s'agir, comme l'ont observé Collin et Kappers (1968) chez Lacerta, de groupes d'axones pinéalofuges ayant quitté l'épiphyse à un ou plusieurs niveaux, pour la longer ensuite dorsalement et rejoindre le tractus pinéal mais elles pourraient correspondre également à des fibres d'un (ou plusieurs) éventuel(s) système(s) efférent(s) (par rapport au système nerveux central), ou pinéalopètes qui innerveraient l'organe pinéal. La mise en évidence de terminaisons axoniques (possédant les carac- 
tères des terminaisons sympathiques) dans l'espace périvasculaire est favorable à la seconde possibilité.

La fonction des cellules interstitielles, qui rappellent les cellules de Müller de la rétine, est délicałe à interpréłer. Leur rôle est cerłainement multiple, mais n'a reçu, jusqu'à ce jour, de preuve expérimentale chez aucun Vertébré :

- leur position dans l'épithélium suggère «qu'elles interviennent dans des phénomènes d'échanges entre le milieu interne et l'organe pinéal » (Collin, 1969) ;

- les caractéristiques ultrastructurales indiquent, chez Esox comme chez de nombreux autres Téléostéens (réf. in Rüdeberg, 1970), une intense activité de synthèse. Comme chez Astyanax (Omura, 1975 ; Herwig, 1976), un produił de synthèse s'accumule ici dans les citernes ergastoplasmiques. Mais la nature (vraisemblablement protéique et/ou glycoprotéique) et le rôle de cetfe substance ne sont encore précisés. Les études ultérieures devront montrer s'il s'agit d'un ralentissement dans le transport réticulaire de substances néosynthétisées, ou de l'accroissement des synthèses dans des conditions particulières. De plus, la signification physiologique de tels composés ne pourra être établie que par une combinaison des recherches cytochimiques et biochimiques ;

- comme chez Carassius (Takahashi, 1969), Phoxinus (Oksche et Kirschstein, 1971) et Astyanax (Omura, 1975 ; Herwig, 1976), les CIE, chez Esox, assurent en partie la digestion des structures en dégénérescence et l'hypothèse d'une synthèse active d'hydrolases stockées partiellement dans les citernes est cohérente avec ce processus ;

- Rüdeberg (1970) est favorable au passage des produits élaborés par les CTP dans les CIE, avant d'atteindre les capillaires. Ceci est vraisemblable dans la mesure où peu de pédicules de CTP entrent en contact avec la lamelle basale (voir Falcon, 1979) ; - les $\mathrm{ClE}$ interviennent également dans une partie du méłabolisme des indolamines (Falcon, Juillard et Collin, en préparation).

A côté des CTP, des CIE et des NS, un 4e type cellulaire $(=\mathrm{CII})$ est parfois identifié dans l'organe pinéal des Téléostéens (Astyanax : Herwig, 1976) et des Batraciens (Diemictylus : Flight, 1973). Collin (1969) a même indiqué l'existence de 5 types cellulaires chez Lampetra. Les Cll (non observées par Owman et Rüdeberg, 1970, chez Esox), présentent de grandes analogies avec certaines cellules de l'organe pinéal de Scyliorhinus (Rüdeberg, 1969) : comme ces dernières (1) elles sont orientées parallèlement à la lamelle basale, (2) elles sont localisées dans le 1/3 basal de l'épithélium épiphysaire, (3) les inclusions observées dans l'un et l'autre cas sont de faille ef de forme identique.

Pour Rüdeberg (1969), il s'agit d'une 2e catégorie de CIE « spécialisées». En l'absence de données sur l'ontogenèse, nous pensons néanmoins qu'elles constituent un 4 e type cellulaire bien individualisé qui présente des caractéristiques morphologiques et cytologiques différentes (par rapport aux $\mathrm{CIE}$, cf. résultats). Le rôle de ces cellules est difficile à élucider en l'absence de résultats sur un éventuel cycle annuel d'évolution des types cellulaires. Sont-elles homologues des cellules à corps résiduels, observées chez Lampetra (Collin, 1969) ou des cellules phagocytantes de l'épithélium pinéal d'Astyanax (Herwig, 1976) ou de Diemictylus (Flight, 1973) ? Selon Flight (1973), Herwig (1976), les cellules du $4 \mathrm{e}$ type ne seraient pas des constituants intrinsèques de l'épithélium épiphysaire. Il s'agirait de cellules microgliales qui pénétreraient par la 
partie périvasculaire de l'épithélium (Herwig, 1976) et se transformeraient en « macrophages » après avoir atteint la lumière épiphysaire (Flight, 1973).

Si les cellules observées chez Esox ef celles de Lampetra, Astyanax ef Diemictylus éfaient homologues, les différences observées en microscopie électronique suggéreraient simplement des états fonctionnels différents. Les inclusions dans les $\mathrm{Cll}$, chez Esox, pourraient stocker des enzymes lytiques complétant la digestion des éléments en dégénérescence déjà observée dans les CIE. II n'est pas exclu que l'activité de CII varie en fonction de l'âge des animaux ef de la période du cycle annuel. Chez les individus que nous avons étudiés, les Cll ne présentent ni vacuoles digestives, ni corps résiduels.

En conclusion, l'organe pinéal du Brochet présente de nombreuses adaptations à la photoréception. Il apparaît comme une rétine simplifiée :

- directe pour le feuillet ventral de l'épithélium où l'énergie lumineuse peut atteindre directement le segment externe des photorécepteurs,

- inverse, pour le feuillet dorsal où la lumière doit traverser l'épithélium de type sensoriel avant d'atteindre le segment externe.

Toutes les dispositions intermédiaires se présentent sur les faces latérales.

Les seules données anatomiques et cytologiques ne permettent pas de définir une activité sécrétoire (neuroendocrinienne) et en conséquence de savoir si l'épiphyse d'Esox dans sa totalité ou si seulement une région particulière est spécialisée dans cette fonction. L'étude ultérieure approfondie des cellules de type photorécepteur ef des neurones sensoriels dans les différentes régions de l'organe et celle des sites cellulaires du métabolisme indolique devraient nous permettre de préciser si à l'apparente "zonation » morphologique correspond réellement une « zonation » fonctionnelle.

Reçu en novembre 1978.

Accepté en décembre 1978.

Remerciements. - Je remercie M. le Prof. J.-P. Collin et $M^{\text {lle }}$ le Dr Juillard, pour les nombreux conseils ef le soutien qu'ils m'ont apportés. Ce travail a pu être réalisé grâce à une bourse et à un contrat DGRST no 76.7.0043 et à l'appui financier du CNRS. Je remercie Mlle $D$. Decourt qui a assuré la dactylographie de ce mémoire.

\section{Références}

BERGMANN G., 1971. Elektronenmikroskopische Untersuchungen am Pinealorgan von Pterophyllum scalore Cuv. et Val. (Cichlidae, Teleostei). Z. Zellforsch., 119, 257-288.

BREUCKER H., HORSTMANN E., 1965. Electronenmikroskopische Untersuchungen am Pinealorgan der Regenbogenforelle (Salmo irideus). Prog. Brain Res., 10, 259-269.

BUNT A. H., KELLY D. E., 1971. Frog pineal photoreceptor renewal : preliminary observations. Anat. Rec., 171, 99-115.

COLLIN J. P., 1969. Contribution à l'étude de l'organe pinéal. De l'épiphyse sensorielle à la glande pinéale : modalités de transformation et implications fonctionnelles. Ann. Stat. Biol. Besse-enChandesse, Suppl. 1, 1-359.

COLLIN J.P., 1971. Differentiation and regression of the cells of the sensory line in the epiphysis cerebri, 79-125. In WOLSTENHOLME G. E. W., KNIGHT J., The pineal gland, Churchill Livingstone, Edinburg-London. 
COLLIN J. P., 1977. La rudimentation des photorécepteurs dans l'organe pinéal des Vertébrés, 393-407. In RAYNAUD A., Mécanismes de la rudimentation des organes chez les embryons de Vertébrés, Coll. int. CNRS no 266, Paris.

COLLIN J. P., ARIËNS KAPPERS J., 1968. Electron microscopic study of pineal innervation in lacertilians. Brain Res., 11, 85-106.

DODT E., 1963. Photosensitivity of the pineal organ in the teleost, Salmo irideus (Gibbons). Experientia, 19, 642 .

FALCON J., 1979. L'épiphyse du brochet (Esox lucius L.). II. Ełude en microscopie électronique de la différenciation et de la rudimentation partielle des photorécepteurs ; conséquences possibles sur l'élaboration des messages photosensoriels. Ann. Biol. anim. Bioch. Biophys. (in press).

FALCON J., MOCQUARD J. P., 1979. L'épiphyse du brochet (Esox lucius L.). III. Voies intrapinéales de conduction des messages photosensoriels. Ann. Biol. anim. Bioch. Biophys. (in press).

FENWICK J. C., 19700. Effect of pinealectomy and bilateral enucleation on the phototactic response and on the conditioned response to light of the goldfish, Carassius auratus L. Can. J. Zool., 48, 175-182.

FENWICK J. C., 1970b. The pineal organ : photoperiod and reproductive cycles in the goidfish Carassius auratus L., J. Endocr., 46, 101-111.

FENWICK J. C., 1970c. Demonstration and effect of melatonin in fish. Gen. comp. Endocr., 14, 86-97.

FLIGHT W. F. G., 1973. Observations on the pineal ultrastructure of the urodele, Diemictylus viridescens. viridescens. Koninkl. Nederl. Akod. Wefensch. Amsferdam Ser. C, 76, 425-448.

HAFEEZ M. A., 1970. Effect of melatonin on body coloration and spontaneous swimming activity in rainbow trout, Salmo gairdneri. Comp. Biochem. Physiol., 36, 639-656.

HAFEEZ M. A., 1971. Light microscopic studies on the pineal organ in teleost fishes with special regard to its function. J. Morph., 134, 281-314.

HAFEEZ M. A., FORD P., 1967. Histology and histochemistry of the pineal organ in the sockeye Salmon, Oncorhyncus nerka, Walbaum. Can. J. Zool., 45, 117-126.

HAFEEZ M. A., QUAY W. B., 1969. Histochemical and experimental studies of 5-hydroxytryptamine in pineal organs of teleosts (Salmo gairdneri and Atherinopsis californiensis). Gen. comp. Endocr., 13, 211-217.

HAFEEZ M. A., QUAY W. B., 1970a. The role of pineal organ in the control of phoiotaxis and body coloration in rainbow trout (Salmo gairdneri, Richardson). Z. vergl. Physiol., 68, 403-416.

HAFEEZ M. A., QUAY W. B., 1970b. Pineal acetylserotonin methyl-transferase activity in the teleost fishes. Hesperoleucus symmefricus and Salmo gairdneri, with evidence for lack of effect of constant light and darkness. Comp. gen. Pharmac., 1, 257-262.

HAFEEZ M. A., ZERIHUN L., 1974. Studies on central projections of the pineal nerve tract in rainbow trout, Salmo goirdneri, Richardson, using cobalt chloride iontophoresis. Cell Tiss. Res., 154, 485-510.

HAFEEZ M. A., ZERIHUN L., 1976. Autoradiographic localization of ${ }^{3} \mathrm{H}-5 \mathrm{HTP}$ and ${ }^{3} \mathrm{H}-5 \mathrm{HT}$ in the pineal organ and circumventricular areas in the rainbow trout, Salmo gairdneri, Richardson. Cell Tiss. Res., 170, 61-76.

HAFEEZ M. A., MERHIGE M. E., 1977. Light and electron microscopic study on the pineal complex of the coelacanth, Latimeria chalumnae, Smith. Cell Tiss. Res., 178, 249-265.

HANYU I., NIWA H., TAMURA T., 1969. A slow potential from the epiphysis cerebri of fishes. Vision Res., 9, 621-623.

HANYU I., NIWA H., 1970. Pineal photosensitivity in three teleosts Salmo irideus, Plecoglossus altivelis and Mugil cephalus. Rev. Con. Biol., 29, 133-140.

HARTWIG H. G., PFAUTSCH M., 1973. Rasterelektronenmikroskopische Beobachtungen an pinealen Sinneszellen der Forelle, Salmo gairdneri (Teleostei). Z. Zellforsch., 138, 585-589.

HERWIG H. J., 1976. Comparative ultrastructural investigations on the pineal organ of the blind cave fish, Anoptichthys jordani, and its ancestor, the eyed river fish, Astyonax mexicanus. Cell Tiss. Res., 167, 297-324.

LUFT J. H., 1961. Improvements in epoxy resins embedding methods. J. biophys. biochem. Cytol., 9 , 409-414.

MARTOJA R., MARTOJA M., 1967. Initiation aux techniques d'hisiologie animale. Masson et Cie, Paris, 345 PP. 
MORITA Y., 1966. Entladungsmuster pinealer Neurone der Regenbogenforelle (Salmo irideus) bei Belichtung des Zwischenhirns. Pflügers Arch. ges. Physiol., 289, 155-167.

MORITA Y., BERGMANN G., 1971. Physiologische Untersuchungen und weitere Bemerkungen zur Struktur des lichtempfindlichen Pinealorgans von Pterophyllum scalare, Cuv. et Val. (Cichlidae, Teleostei). Z. Zellforsch., 119, 289-294.

MURPHY R. C., 1971. The structure of the pineal organ of the bluefin tuna, Thunnus thynnus. J. Morph., 133, 1-16.

MCNULTY J. A., 1976. A comparative study of the pineal complex in the deep sea fishes Bathylagus wesethi and Nezumia liolepsis. Cell Tiss. Res., 172, 205-225.

NCNULTY J. A., NAFPAKTITIS B. G., 1976. The structure and development of the pineal complex in the lanternfish Triphoturus mexicanus (family Myctophidae). J. Morph., 150, 579-605.

OGURI M., OMURA Y., HIBIYA T., 1968. Uptake of ${ }^{14} \mathrm{C}$-labelled-5-hydroxytryptophan into the pineal organ of rainbow trout. Bull. Jap. Soc. Sci. Fish., 34, 687-690.

OKSCHE A., KIRSCHSTEIN H., 1967. Die Ultrastrukfur der Sinneszellen im Pinealorgan von Phoxinus laevis L., Z. Zellforsch., 78, 151-166.

OKSCHE A., KIRSCHSTEIN H., 1971. Weitere elektronenmikroskopische Untersuchungen am Pinealorgan von Phoxinus laevis (Teleostei, Cyprinidae). Z. Zellforsch., 112, 572-588.

OMURA Y., 1975. Influence of light and darkness on the ultrastructure of the pineal organ of the blind cave fish, Astyanax mexicanus. Cell. Tiss. Res., 160, 99-112.

OMURA Y., OGURI M., 1969. Histological studies on the pineal organ of 15 species of teleosts. Bull. Jap. Soc. Sci. Fish., 35, 991-1000.

OMURA Y., KITOH J., OGURI M., 1969. The photoreceptor cell of the pineal organ of the ayu, Plecoglossus altivelis. Bull. Jap. Soc. Sci. Fish., 35, 1067-1071.

OMURA Y., OGURI M., 1971. The development and degeneration of the photoreceptor outer segment of the fish pineal organ. Bull. Jap. Soc. Sci. Fish., 37, 851-860.

OWMAN C., RUDEBERG C., 1970. Light, fluorescence and electron microscopic studies on the pineal organ of the pike Esox lucius, with special regard to 5-hydroxytryptamine. Z. Zellforsch., 107, 522-55.

QUAY W. B., 1965. Retinal and pineal hydroxyindole-O-methyl transferase activity in vertebrates. Life Sci., 4, 983-991.

QUAY W. B., 1974. Pineal chemistry. Charles C. Thomas, Springfield, 430 pP.

REYNOLDS E. S., 1963. The use of lead citrate at high $\mathrm{pH}$ an as electron opaque stain in electron microscopy. J. Cell Biol., 17, 208-212.

RÜDEBERG C., 1966. Electron microscopical observations on the pineal organ of the teleosts Mugil auratus (Risso) and Uranoscopus scaber (Linné). Pubbl. Staz. Zool. Napoli, 35, 47-60.

RÜDEBERG C., 1968. Structure of the pineal organ of the sardine, Sardina pilchardus sardina (Risso), and some further remarks on the pineal organ of Mugil spp. Z. Zellforsch., 84, 219. 237.

RÜDEBERG C., 1969. Light and electron microscopic studies on the pineal organ of the dogfish, Scyliorhinus canicula L., Z. Zellforsch., 96, 548-581.

RÜDEBERG C., 1970. Light and electron microscopic investigations on the pineal and parapineal organs of fishes. Thesis, Lund, $29 \mathrm{pP}$.

RÜDEBERG C., 1971. Structure of the pineal organs of Anguilla anguilla, L. and Lebistes reticulatus, Peters (Teleostei). Z. Zellforsch., 122, 227-243.

SMITH J. R., WEBER L. J., 1976a. Alterations in diurnal pineal hydroxyindole-O-methyltransferase (HIOMT) activity in steelhead trout (Salmo gairdneri) associated with changes in environmental background color. Comp. Biochem. Physiol., 53C, 33-35.

SMITH J. R., WEBER L. J., 1976b. The regulation of day-night changes in hydroxyindole-O-methyltransferase activity in the pineal gland of the steelhead trout (Salmo gairdneri). Con. J. Zool., 54, 1530-1534.

STUDNICKA F. D., 1905. Die Parietalorgane, 1-254. In OPPEL A., Lehrbuch der vergleichenden mikroskopischen Anatomie der Wirbeltiere. V, G. Fischer, Jena.

TABATA M., TAMURA T., NIWA H., 1975. Origin of the slow potential in the pineal organ of the rainbow trout. Vision Res., 15, 737-740.

TAKAHASHI H., 1969. Light and electron microscopic studies on the pineal organ of the goldfish, Carassius auralus L., Bull. Fac. Fish., Hokkaido Univ., 20, 143-157. 
TAKAHASHI H., KASUGA S., 1971. Fine structure of the pineal organ of the medaka, Oryzias latipes. Bull. Fac. Fish., Hokkaido Univ., 22, 1-10.

URASAKI H., 1976a. Fine organization of nervous system and sensory cell in the pineal gland of the teleost, Oryzias latipes. Bull. Lib. Arts ef Sci. Course, Sch. Med. Nihon Univ., 4, 15-20.

URASAKI H., 1976b. The role of pineal and eyes in the photoperiodic effect on the gonad of the medaka, Oryzios latipes. Chronobiologia, 3, 228-234.

VLAMING V. L. de, SAGE M., CHARLTON C. B., 1974. The effects of melatonin treatment and gonosomatic index in the teleost, Fundulus similis, and the tree frog, Hyla cinerea. Gen. comp. Endocr., 22, 433-438.

WATSON M. L., 1958. Staining of tissue sections for electron microscopy with heavy metals. J. biophys. biochem. Cytol., 4, 475-478. 